\title{
MICROFLORA FÚNGICA DE SEMENTES DE MILHO EM AMBIENTES DE ARMAZENAMENTO'
}

\author{
Maria Aparecida de Souza Tanaka ${ }^{2,4 *}$; Jocely Andreuccetti Maeda ${ }^{3,4}$; Isabel Helena de Almeida \\ Zeituni Plazas ${ }^{3,5}$ \\ ${ }^{2}$ Centro de Fitossanidade - IAC, C.P. 28 - CEP: 13001-970 - Campinas, SP. \\ ${ }_{4}^{3}$ Centro de Produção de Material Propagativo - IAC. \\ ${ }^{4}$ Bolsista CNPq. \\ ${ }^{5}$ Bolsista FAPESP. \\ Autor correspondente < matanaka@cec.iac.br >
}

\begin{abstract}
RESUMO: Durante o armazenamento, vários fungos podem permanecer associados às sementes de milho, causando deterioração ou se mantendo viáveis, infectando posteriormente a plântula. Objetivou-se neste trabalho verificar a sobrevivência de fungos associados às sementes de milho durante 12 meses de armazenamento, em câmara fria $\left(14^{\circ} \mathrm{C} ; 40 \%\right.$ UR) e em ambiente não controlado. Observaram-se com maior freqüência os fungos de campo Alternaria alternata, Bipolaris maydis, Cephalosporium acremonium, Cladosporium herbarum, Fusarium moniliforme e Rhizoctonia solani, além de Rhizopus spp. e Trichoderma spp., cuja sobrevivência decresceu ao longo do armazenamento, de modo muito mais acentuado em condição de ambiente não controlado, em comparação à câmara fria. Aspergillus e Penicillium tiveram suas incidências aumentadas ao longo do período, principalmente em ambiente não controlado. Nessa condição, a sobrevivência de Fusarium moniliforme foi reduzida gradativamente até o final dos doze meses; comparativamente, em câmara fria, a viabilidade do fungo foi menos afetada. Bipolaris maydis manteve-se viável na maioria dos lotes, durante todo o armazenamento em câmara fria; em ambiente não controlado, o fungo sobreviveu durante quatro a dez meses, dependendo do lote avaliado. O armazenamento em ambiente não controlado, embora tenha provocado a redução do inóculo de F. moniliforme e outros fungos importantes, poderia acelerar o processo de deterioração das sementes. Em câmara fria, por outro lado, a viabilidade dos fungos é favorecida, comprometendo a qualidade sanitária das sementes.
\end{abstract}

Palavras-chave: Zea mays, patologia de sementes, armazenamento, fungos

\section{FUNGI ASSOCIATED TO CORN SEEDS UNDER STORAGE CONDITIONS}

\begin{abstract}
During storage, several kinds of fungi can remain associated to corn seeds, either causing their deterioration or simply remaining viable to infect germinating seedlings. The objective of this work was to evaluate the survival of fungi in corn seeds stored for twelve months in a cold chamber $\left(14^{\circ} \mathrm{C}\right.$ and $\left.40 \% \mathrm{RH}\right)$ or stored under uncontrolled conditions. A larger frequency of Alternaria alternata, Bipolaris maydis, Cephalosporium acremonium, Cladosporium herbarum, Fusarium moniliforme, Rhizoctonia solani, Rhizopus spp. and Trichoderma spp. occurred in the cold condition. The survival decreased with the storage period mainly under uncontrolled atmosphere as compared to the cold environment. The incidence of Aspergillus and Penicillium increased with the storage, mainly under the uncontrolled ambient. Fusarium moniliforme, on the other hand, decreased sharply under ambiental conditions at the end of the period. In the cold chamber, the F. moniliforme viability was less affected. Bipolaris maydis remained viable in most of the seed lots during the entire storage period in the cold chamber. Under uncontrolled conditions, this fungus survived for four to twelve months, depending on the lot studied. The seed storage under uncontrolled ambient may reduce the inoculum of $F$. moniliforme and other important fungi for the maize crop, but this condition may accelerate seed deterioration. In the cold condition, otherwise, the ambient is favorable to preserve the physiological quality of the seeds, but the maintenance of fungi viability may reduce their sanitary quality.

Key words: Zea mays, seed pathology, storage, fungi
\end{abstract}

\section{INTRODUÇÃO}

As sementes de milho podem ser invadidas por vários fungos, desde a sua formação, durante o seu desenvolvimento e também após a colheita, no armazenamento. Dessa maneira, as sementes podem permitir a sobrevivência e veiculação de patógenos, bem como a proliferação de fungos de armazenamento, responsáveis pela sua deterioração (Tuite \& Forster, 1979; Luca Filho, 1987; Fernandes \& Oliveira, 1997).

O armazenamento das sementes para fins agrícolas geralmente é utilizado para a manutenção de estoques no período da entressafra ou para a provisão de quantidades suficientes para atender a demanda de

${ }^{1}$ Trabalho apresentado no $10^{\circ}$ Congresso Brasileiro de Sementes, Foz do Iguaçu, 1997. 
comercialização. Muitas vezes é necessário o armazenamento por longos períodos, para garantir estoques em anos que sucedem frustrações de safras, quando as sementes produzidas estão aquém do padrão exigido, ou para a conservação de germoplasma (Berjak, 1987b; Wetzel, 1987). No entanto, as mesmas condições de armazenamento que permitem a manutenção da viabilidade das sementes, podem também favorecer a sobrevivência de muitos patógenos importantes para a cultura.

Os fungos presentes nas sementes armazenadas são tradicionalmente divididos em dois grupos: de campo e de armazenamento. Os primeiros, invadem as sementes ainda no campo, requerendo para o seu crescimento, umidade relativa em torno de $90-95 \%$. O tempo de sobrevivência desses fungos nas sementes está diretamente relacionado com as condições de ambiente do armazém (Lal \& Kapoor, 1979; Berjak, 1987a; Meronuck, 1987). Os fungos de armazenamento, por sua vez, estão presentes nas sementes recém-colhidas, geralmente em porcentagens muito baixas. São capazes de sobreviver em ambiente com baixa umidade, proliferando em sucessão aos fungos de campo e causando a deterioração das sementes (Berjak, 1987a; Wetzel, 1987; Carvalho \& Nakagawa, 1988).

Dentre os fungos de campo veiculados pelas sementes de milho, no Brasil, Fusarium moniliforme é o mais freqüente (Reis et al., 1995; Peixoto et al., 1998; Goulart \& Fialho, 1999). Vários outros fungos, podendose citar Bipolaris maydis, Cephalosporium acremonium, Colletotrichum graminicola, Curvularia spp., Diplodia maydis, Drechslera spp., Epicoccum spp., Nigrospora oryzae, Rhizoctonia solani e Trichoderma spp., também são comumente detectados em associação com as sementes de milho. Quanto aos fungos de armazenamento, os mais freqüentes geralmente são Aspergillus spp. e Penicillium spp. (Tuite et al., 1985; Luz, 1995; Pinto, 1998).

A literatura disponível sobre a natureza dos fungos de armazenamento presentes em sementes de milho e os danos por eles provocados é relativamente vasta. No entanto, pouco se conhece a respeito do comportamento dos fungos fitopatogênicos (de campo), nas sementes armazenadas, principalmente se conservadas em diferentes condições de ambiente, as quais podem afetar diretamente a sua sobrevivência (Lal \& Kapoor, 1979; Meronuck, 1987; Halfon-Meiri \& Solel, 1990).

Tendo como objetivo acrescentar mais informações sobre esse assunto, neste trabalho pretendeuse verificar a sobrevivência de fungos associados às sementes de milho durante doze meses de armazenamento, em câmara fria e em ambiente não controlado.

\section{MATERIAL E MÉTODOS}

O trabalho foi realizado no Centro de Fitossanidade, localizado no Núcleo Experimental do Instituto Agronômico, em Campinas, SP.
Foram utilizadas amostras de sementes fiscalizadas de milho híbrido da cultivar G-85, produzidas na safra 93/94, pertencentes a oito lotes que apresentavam diferentes incidências de fungos. As sementes foram embaladas em sacos de papel kraft multifoliado e armazenadas, no período de junho de 1994 a junho de 1995 , em câmara fria (temperatura de $14^{\circ} \mathrm{C}$ e UR $40 \%$ ) e em condições ambientes de laboratório, onde a temperatura ficou situada na faixa de 18 a $32^{\circ} \mathrm{C}$ e a umidade relativa do ar entre 65 e $95 \%$.

Em intervalos bimestrais foi retirada uma amostra de 200 sementes, para ser avaliada quanto à sanidade. O método utilizado foi o de papel de filtro com congelamento, tendo este procedimento a finalidade de impedir a germinação e facilitar o exame das sementes (Tempe, 1970; Machado, 1988). As sementes foram colocadas eqüidistantes entre si, em placas de Petri de $9 \mathrm{~cm}$ de diâmetro (10 sementes/placa), contendo duas folhas de papel de filtro umedecidas com água destilada. Em seguida, foram mantidas durante 24 horas em temperatura de $20-22^{\circ} \mathrm{C}$ e fotoperíodo de 12 / 12 horas de escuro e luz fluorescente branca de $40 \mathrm{~W}$, posicionada a $40 \mathrm{~cm}$ acima das placas e $20 \mathrm{~cm}$ entre si. Após, as sementes foram submetidas ao congelamento durante 24 horas, retornando à sala de incubação anteriormente descrita, onde permaneceram mais cinco dias. A detecção dos fungos associados às sementes foi realizada em estereomicroscópio e, quando necessário, foram examinadas lâminas ao microscópio composto.

$\mathrm{O}$ experimento foi instalado em delineamento inteiramente casualizado, sendo que, para cada lote houve 14 tratamentos, constituídos de dois ambientes de armazenamento e sete épocas de amostragem, em arranjo fatorial, com quatro repetições de 50 sementes por tratamento. Os dados obtidos foram submetidos à análise estatística, sendo as médias comparadas pelo teste de Tukey a $5 \%$.

\section{RESULTADOS E DISCUSSÃO}

Os fungos Alternaria alternata, Aspergillus spp., Bipolaris maydis (Helminthosporium maydis), Cephalosporium acremonium, Cladosporium herbarum, Fusarium moniliforme, Penicillium spp., Rhizopus spp. e Trichoderma spp. foram os mais freqüentemente observados. Por outro lado, Curvularia spp., Colletotrichum graminicola, Fusarium spp., Diplodia maydis, Epicoccum spp., Gonatobotrys spp., Phoma spp. e Rhizoctonia solani ocorreram com menor freqüência, e em alguns lotes (TABELAS 1 a 8). A incidência de Rhizopus spp. foi baixa, superando $20 \%$ apenas no lote 3 (TABELA 3).

De maneira geral, durante os doze meses de armazenamento a sobrevivência dos fungos considerados de campo foi decrescendo, de modo muito mais acentuado em condição de ambiente, em comparação à câmara fria. Esses dados confirmaram os resultados obtidos por Oliveira et al. (1997) que, 
TABELA 1 - Porcentagens de fungos detectados em sementes de milho do lote 1, armazenadas durante 12 meses em câmara fria e em condição ambiente.

\begin{tabular}{|c|c|c|c|c|c|c|c|c|c|}
\hline \multirow{2}{*}{ Fungo } & \multirow{2}{*}{ Local armaz. } & \multicolumn{7}{|c|}{ Mês de armazenamento } & \multirow{2}{*}{ Média } \\
\hline & & 0 & 2 & 4 & 6 & 8 & 10 & 12 & \\
\hline \multirow[t]{2}{*}{ Alternaria alternata } & $\mathrm{CF}^{1}$ & 3 aA & $2 \mathrm{abA}$ & $2 \mathrm{abA}$ & $1 \mathrm{bA}$ & $2 \mathrm{abA}$ & $1 \mathrm{bA}$ & $1 \mathrm{bA}$ & $1,71 \mathrm{~A}$ \\
\hline & $\mathrm{Amb}^{2}$ & $3 a b A$ & $2 \mathrm{abA}$ & $4 \mathrm{aA}$ & $2 a b A$ & $2 a b A$ & $1 \mathrm{bA}$ & $1 \mathrm{bA}$ & $2,14 \mathrm{~A}$ \\
\hline \multirow[t]{2}{*}{ Aspergillus spp. } & CF & $7 \mathrm{abA}$ & $5 \mathrm{bA}$ & $5 \mathrm{bA}$ & $5 \mathrm{bB}$ & 9 aA & $8 \mathrm{abB}$ & $9 \mathrm{aB}$ & $6,86 \mathrm{~B}$ \\
\hline & Amb & $7 \mathrm{dA}$ & $6 \mathrm{dA}$ & $6 \mathrm{dA}$ & $10 \mathrm{cA}$ & $9 \mathrm{cA}$ & $13 \mathrm{bA}$ & $15 \mathrm{aA}$ & $9,43 \mathrm{~A}$ \\
\hline \multirow[t]{2}{*}{ Bipolaris maydis } & $\mathrm{CF}$ & $4 \mathrm{aA}$ & $4 \mathrm{aA}$ & $3 a b A$ & $2 \mathrm{cA}$ & $2 \mathrm{cA}$ & $2 \mathrm{cA}$ & $2 \mathrm{cA}$ & $2,71 \mathrm{~A}$ \\
\hline & Amb & $4 \mathrm{aA}$ & $2 \mathrm{bA}$ & $1 \mathrm{bA}$ & $0 \mathrm{bA}$ & $0 \mathrm{bA}$ & $0 \mathrm{bA}$ & $0 \mathrm{bA}$ & $1,14 \mathrm{~A}$ \\
\hline \multirow{2}{*}{ Cephalosporium acremonium } & $\mathrm{CF}$ & $29 \mathrm{aA}$ & $27 \mathrm{abA}$ & $28 a b A$ & $25 \mathrm{bcA}$ & $22 \mathrm{cA}$ & $17 \mathrm{dA}$ & 11 eA & $22,71 \mathrm{~A}$ \\
\hline & Amb & 29 aA & $25 \mathrm{aA}$ & $18 \mathrm{bB}$ & $5 \mathrm{cB}$ & $3 \mathrm{cB}$ & $4 \mathrm{cB}$ & $3 \mathrm{cB}$ & $12,43 \mathrm{~B}$ \\
\hline \multirow[t]{2}{*}{ Cladosporium herbarum } & $\mathrm{CF}$ & $3 \mathrm{cdA}$ & $6 \mathrm{aA}$ & 6 aA & $4 \mathrm{bcA}$ & $2 d A$ & $4 \mathrm{bcA}$ & $5 \mathrm{abA}$ & $4,29 \mathrm{~A}$ \\
\hline & Amb & $3 \mathrm{bcA}$ & $6 \mathrm{aA}$ & $4 \mathrm{bA}$ & $3 \mathrm{bcA}$ & $3 \mathrm{bcA}$ & $2 \mathrm{~cd} A$ & $1 \mathrm{~dB}$ & $3,14 \mathrm{~A}$ \\
\hline \multirow[t]{2}{*}{ Curvularia spp. } & $\mathrm{CF}$ & 0 & 2 & 0 & 0 & 1 & 0 & 1 & 0,57 \\
\hline & Amb & 0 & 0 & 0 & 0 & 0 & 0 & 0 & 0,00 \\
\hline \multirow[t]{2}{*}{ Diplodia maydis } & $\mathrm{CF}$ & 0 & 1 & 0 & 1 & 1 & 0 & 0 & 0,42 \\
\hline & Amb & 0 & 0 & 0 & 0 & 1 & 0 & 0 & 0,14 \\
\hline \multirow[t]{2}{*}{ Epicoccum spp. } & $\mathrm{CF}$ & 0 & 1 & 0 & 2 & 0 & 0 & 1 & 0,57 \\
\hline & Amb & 0 & 0 & 0 & 0 & 2 & 0 & 0 & 0,28 \\
\hline \multirow[t]{2}{*}{ Fusarium moniliforme } & $\mathrm{CF}$ & $59 a b A$ & $52 \mathrm{cA}$ & $58 \mathrm{bA}$ & $60 \mathrm{abA}$ & $61 \mathrm{abA}$ & $62 \mathrm{aA}$ & $60 \mathrm{abA}$ & $58,86 \mathrm{~A}$ \\
\hline & Amb & 59 aA & $57 \mathrm{aA}$ & $36 \mathrm{bB}$ & $25 \mathrm{cB}$ & $20 \mathrm{~dB}$ & $16 \mathrm{~dB}$ & $5 \mathrm{eB}$ & $31,14 \mathrm{~B}$ \\
\hline \multirow[t]{2}{*}{ Gonatobotrys spp. } & $\mathrm{CF}$ & $2 \mathrm{aA}$ & $1 \mathrm{aA}$ & $2 \mathrm{aA}$ & $2 \mathrm{aA}$ & $1 \mathrm{aA}$ & $1 \mathrm{aA}$ & $2 \mathrm{aA}$ & $1,57 \mathrm{~A}$ \\
\hline & Amb & $2 a b A$ & $2 a b A$ & $3 \mathrm{aA}$ & $1 \mathrm{bA}$ & $0 \mathrm{cA}$ & $0 \mathrm{cA}$ & $0 \mathrm{cB}$ & $1,29 \mathrm{~A}$ \\
\hline \multirow[t]{2}{*}{ Penicillium spp. } & $\mathrm{CF}$ & $25 \mathrm{cA}$ & 26 bcA & $24 \mathrm{cA}$ & $32 \mathrm{aA}$ & $31 \mathrm{aA}$ & $30 \mathrm{abB}$ & $32 \mathrm{aB}$ & 28,57 B \\
\hline & Amb & $25 \mathrm{eA}$ & $28 \mathrm{de} A$ & 24 eA & $30 \mathrm{cdA}$ & $34 \mathrm{cA}$ & $47 \mathrm{bA}$ & $53 \mathrm{aA}$ & $34,43 \mathrm{~A}$ \\
\hline \multirow[t]{2}{*}{ Phoma spp. } & CF & 2 & 0 & 0 & 0 & 0 & 0 & 0 & 0,29 \\
\hline & Amb & 2 & 1 & 1 & 0 & 0 & 0 & 0 & 0,57 \\
\hline \multirow[t]{2}{*}{ Rhizopus spp. } & $\mathrm{CF}$ & $0 \mathrm{bA}$ & $0 \mathrm{bA}$ & $0 \mathrm{bB}$ & $2 \mathrm{bA}$ & $0 \mathrm{bA}$ & $2 \mathrm{bA}$ & $7 \mathrm{aA}$ & $1,57 \mathrm{~A}$ \\
\hline & Amb & $0 \mathrm{cA}$ & $0 \mathrm{cA}$ & $6 \mathrm{aA}$ & $3 \mathrm{bcA}$ & $0 \mathrm{cA}$ & $0 \mathrm{cA}$ & $0 \mathrm{cB}$ & $1,29 \mathrm{~A}$ \\
\hline \multirow[t]{2}{*}{ Trichoderma spp. } & $\mathrm{CF}$ & 0 & 0 & 0 & 0 & 2 & 2 & 5 & 1,29 \\
\hline & Amb & 0 & 0 & 0 & 0 & 0 & 0 & 0 & 0,00 \\
\hline
\end{tabular}

${ }^{1} \mathrm{CF}=$ Câmara fria; ${ }^{2} \mathrm{Amb} .=$ Ambiente. Letras minúsculas iguais nas linhas e maiúsculas nas colunas, para cada fungo, não apresentam diferença estatística (Tukey $5 \%$ ).

armazenando sementes de milho em ambiente convencional, também observaram redução da incidência de Fusarium moniliforme e Cephalosporium sp.

Fusarium moniliforme, um dos principais patógenos associados às sementes de milho, teve a sua sobrevivência bastante reduzida durante 0 armazenamento em ambiente não controlado (TABELAS 1 a 8). Em câmara fria, no entanto, a viabilidade do fungo foi menos afetada. Tal fato pode ser bem visualizado na TABELA 6 , para o lote 6 , em que a incidência de $F$. moniliforme, inicialmente de $84 \%$, ainda era de $70 \%$ ao final de 12 meses, em câmara fria; em condição de ambiente, para este mesmo lote, a incidência caiu gradativamente, até $14 \%$ ao final do período. Tendo em vista a importância da associação de F. moniliforme com as sementes de milho na redução do estande, conforme salientado por vários autores, como Ochor et al. (1987); Bacon et al. (1994) e Casa et al. (1998), infere-se que a conservação das sementes em câmara fria, com o intuito de preservar a sua qualidade fisiológica, pode, ao mesmo tempo, estar favorecendo a sobrevivência do fungo. Conforme Tanaka (1976), Tanaka \& Balmer (1980) e Casa et al. (1995), principalmente se a semeadura for efetuada em solos com temperaturas sub-ótimas para a germinação e estabelecimento da plântula, este patógeno pode causar prejuízos.

Dentre os demais fungos considerados patogênicos, Bipolaris maydis manteve-se viável em câmara fria durante todo o período de armazenamento, em seis lotes avaliados (TABELAS 1, 3, 4, 5, 7 e 8). Em condição ambiente, o fungo sobreviveu até o décimo mês no lote 8 (TABELA 8), porém não além do quarto mês no lote 1 (TABELA 1). No lote 2, a sobrevivência foi detectada até o sexto mês (TABELA 2), sendo que na maioria dos lotes estudados, não excedeu oito meses (TABELAS 3, 4, 7 e 8). Essas variações podem ser decorrentes dos fatores relacionados com a longevidade dos fungos em associação com as sementes armazenadas, destacando-se, dentre eles, o tipo de inóculo (esporos ou micélio dormente) e a sua localização nas diferentes partes da semente (Berjak, 1987a; Machado, 1988).

Cephalosporium acremonium, cuja ocorrência também é freqüente em sementes de milho, teve sua viabilidade bastante reduzida durante $o$ armazenamento, mesmo em câmara fria (TABELAS 1 a 8). Em ambiente 
TABELA 2 - Porcentagens de fungos detectados em sementes de milho do lote 2, armazenadas durante 12 meses em câmara fria e em condição ambiente.

\begin{tabular}{|c|c|c|c|c|c|c|c|c|c|}
\hline \multirow{2}{*}{ Fungo } & \multirow{2}{*}{ Local armaz. } & \multicolumn{7}{|c|}{ Mês de armazenamento } & \multirow{2}{*}{ Média } \\
\hline & & 0 & 2 & 4 & 6 & 8 & 10 & 12 & \\
\hline \multirow[t]{2}{*}{ Altemaria alternata } & $\mathrm{CF}^{1}$ & $11 \mathrm{aA}$ & $11 \mathrm{aA}$ & $8 a b A$ & $6 \mathrm{bA}$ & $6 \mathrm{bA}$ & $5 \mathrm{bA}$ & $5 \mathrm{bA}$ & $7,43 \mathrm{~A}$ \\
\hline & $\mathrm{Amb}^{2}$ & $11 \mathrm{aA}$ & $7 \mathrm{bB}$ & $4 \mathrm{cB}$ & $4 \mathrm{cA}$ & $3 \mathrm{cA}$ & $3 \mathrm{cA}$ & $4 \mathrm{cA}$ & $5,14 \mathrm{~A}$ \\
\hline \multirow[t]{2}{*}{ Aspergillus spp. } & CF & $13 \mathrm{aA}$ & $13 \mathrm{aA}$ & $14 \mathrm{aA}$ & $13 \mathrm{aB}$ & $10 \mathrm{bB}$ & $13 \mathrm{aB}$ & $13 \mathrm{aB}$ & $12,71 \mathrm{~B}$ \\
\hline & Amb. & $13 \mathrm{dA}$ & $16 \mathrm{dA}$ & $16 \mathrm{dA}$ & $20 \mathrm{cA}$ & $25 \mathrm{abA}$ & $23 \mathrm{bcA}$ & 28 aA & $20,14 \mathrm{~A}$ \\
\hline \multirow[t]{2}{*}{ Bipolaris maydis } & CF & $3 \mathrm{aA}$ & $3 \mathrm{aA}$ & $3 \mathrm{aA}$ & $2 a b A$ & $1 \mathrm{bcA}$ & $1 \mathrm{bcA}$ & $0 \mathrm{cA}$ & $1,86 \mathrm{~A}$ \\
\hline & Amb. & $3 \mathrm{aA}$ & $1 \mathrm{abA}$ & $1 \mathrm{abA}$ & $1 \mathrm{abA}$ & $0 \mathrm{cA}$ & $0 \mathrm{cA}$ & $0 \mathrm{cA}$ & $0,86 \mathrm{~A}$ \\
\hline \multirow[t]{2}{*}{ Cephalosporium acremonium } & CF & $22 \mathrm{aA}$ & $16 \mathrm{~cd} A$ & $18 \mathrm{abcA}$ & $18 a b c A$ & $17 \mathrm{bcA}$ & $12 \mathrm{de} A$ & $8 \mathrm{eA}$ & $15,87 \mathrm{~A}$ \\
\hline & Amb. & $22 \mathrm{aA}$ & $18 \mathrm{abA}$ & $15 \mathrm{bcA}$ & $11 \mathrm{cB}$ & $2 \mathrm{~dB}$ & $2 \mathrm{~dB}$ & $3 \mathrm{~dB}$ & $10,43 \mathrm{~B}$ \\
\hline \multirow[t]{2}{*}{ Cladosporium herbarum } & $\mathrm{CF}$ & $6 \mathrm{bA}$ & $6 \mathrm{bA}$ & $7 \mathrm{abA}$ & $8 \mathrm{aA}$ & $3 \mathrm{cdA}$ & $4 \mathrm{cA}$ & $2 \mathrm{dA}$ & $5,14 \mathrm{~A}$ \\
\hline & Amb. & $6 \mathrm{aA}$ & $4 \mathrm{abA}$ & $4 a b A$ & $4 \mathrm{abB}$ & $3 \mathrm{cA}$ & $3 \mathrm{cA}$ & $3 \mathrm{cA}$ & $3,88 \mathrm{~A}$ \\
\hline \multirow[t]{2}{*}{ Colletotrichum graminicola } & CF & 1 & 1 & 1 & 0 & 1 & 1 & 0 & 0,71 \\
\hline & Amb. & 1 & 1 & 1 & 0 & 0 & 0 & 0 & 0,43 \\
\hline \multirow[t]{2}{*}{ Fusarium moniliforme } & CF & $51 \mathrm{aA}$ & $49 \mathrm{abA}$ & $45 \mathrm{bcA}$ & $42 \mathrm{cdA}$ & $41 \mathrm{cdA}$ & $41 \mathrm{cdA}$ & $40 \mathrm{dA}$ & $44,14 \mathrm{~A}$ \\
\hline & Amb. & $51 \mathrm{aA}$ & $36 \mathrm{bB}$ & $30 \mathrm{bcB}$ & $26 \mathrm{cB}$ & $18 \mathrm{~dB}$ & $16 \mathrm{~dB}$ & $8 \mathrm{eB}$ & $26,43 \mathrm{~B}$ \\
\hline \multirow[t]{2}{*}{ Fusarium spp. } & $\mathrm{CF}$ & 0 & 0 & 0 & 0 & 2 & 0 & 0 & 0,29 \\
\hline & Amb. & 0 & 0 & 0 & 0 & 0 & 0 & 0 & 0,00 \\
\hline \multirow[t]{2}{*}{ Nigrospora spp. } & CF & $1 \mathrm{aA}$ & $0 \mathrm{bB}$ & $1 \mathrm{aA}$ & $1 \mathrm{aA}$ & $0 \mathrm{bA}$ & $0 \mathrm{bA}$ & $0 \mathrm{bA}$ & $0,43 \mathrm{~A}$ \\
\hline & Amb. & $1 \mathrm{cA}$ & $4 \mathrm{abA}$ & $3 \mathrm{bA}$ & $0 \mathrm{dA}$ & $0 \mathrm{dA}$ & $0 \mathrm{dA}$ & $0 \mathrm{dA}$ & $1,14 \mathrm{~A}$ \\
\hline \multirow[t]{2}{*}{ Penicillium spp. } & $\mathrm{CF}$ & $42 \mathrm{aA}$ & $36 \mathrm{bA}$ & 32 bB & $35 \mathrm{bB}$ & $43 \mathrm{aB}$ & $42 \mathrm{aB}$ & $46 \mathrm{aB}$ & $39,43 \mathrm{~B}$ \\
\hline & Amb. & $42 \mathrm{cA}$ & $37 \mathrm{cA}$ & $43 \mathrm{bcA}$ & $49 \mathrm{bA}$ & $56 \mathrm{aA}$ & $59 \mathrm{aA}$ & $61 \mathrm{aA}$ & $49,57 \mathrm{~A}$ \\
\hline \multirow[t]{2}{*}{ Rhizoctonia solani } & $\mathrm{CF}$ & 1 & 0 & 0 & 0 & 0 & 0 & 0 & 0,14 \\
\hline & Amb. & 1 & 0 & 0 & 0 & 0 & 0 & 0 & 0,14 \\
\hline \multirow[t]{2}{*}{ Rhizopus spp. } & $\mathrm{CF}$ & $0 \mathrm{cA}$ & $0 \mathrm{cA}$ & $0 \mathrm{cA}$ & $4 \mathrm{aB}$ & $2 \mathrm{bA}$ & $0 \mathrm{cB}$ & $2 \mathrm{bB}$ & $1,14 \mathrm{~A}$ \\
\hline & Amb. & $0 \mathrm{cA}$ & $0 \mathrm{cA}$ & $0 \mathrm{cA}$ & $8 \mathrm{aA}$ & $0 \mathrm{cB}$ & $5 \mathrm{bA}$ & $5 \mathrm{bA}$ & $2,57 \mathrm{~A}$ \\
\hline \multirow[t]{2}{*}{ Trichoderma spp. } & CF & 0 & 0 & 0 & 0 & 5 & 4 & 9 & 2,57 \\
\hline & Amb. & 0 & 0 & 0 & 0 & 0 & 0 & 0 & 0,00 \\
\hline
\end{tabular}

${ }^{1} \mathrm{CF}=$ Câmara fria; ${ }^{2} \mathrm{Amb} .=$ Ambiente. Letras minúsculas iguais nas linhas e maiúsculas nas colunas, para cada fungo, não apresentam diferença estatística (Tukey $5 \%$ ).

não controlado a perda da viabilidade foi ainda maior, sendo quase total no décimo segundo mês, no lote 5 (TABELA 5).

Colletotrichum graminicola foi constatado apenas nos lotes 2 (TABELA 2) e 6 (TABELA 6), com incidência de $1 \%$. No primeiro lote foi observada incidência até 0 décimo mês de armazenamento em câmara fria e apenas até o quarto mês, em condição de ambiente (TABELA 2).

A ocorrência de Aspergillus spp. foi constante nas sementes de todos os lotes e nas duas condições de armazenamento. A porcentagem de incidência, no entanto, não aumentou consideravelmente com o passar do tempo (TABELAS 1 a 8). Para alguns lotes, em câmara fria, houve tendência de redução da incidência ao final do armazenamento. Tal fato provavelmente resultou do comportamento das espécies de Aspergillus presentes nas sementes, uma vez que existe variação entre as espécies quanto ao período de sobrevivência, em função do teor de água da semente e da umidade relativa do ar (Lal \& Kapoor, 1979).
Penicillium spp., tradicionalmente classificados como fungos de armazenamento, tiveram suas porcentagens de incidência aumentadas em função do tempo em que as sementes foram armazenadas. Em câmara fria, esse aumento da incidência foi menos acentuado.

O armazenamento das sementes de milho durante doze meses em ambiente não controlado, com temperatura de 18 a $32^{\circ} \mathrm{C}$ e umidade relativa de 65 a $95 \%$, pode se traduzir em redução do inóculo de $F$. moniliforme e outros fungos importantes para a cultura; no entanto, os fungos de armazenamento poderiam acelerar o processo de deterioração das sementes, afetando a sua qualidade fisiológica, que seria melhor preservada em câmara fria $\left(14^{\circ} \mathrm{C}\right.$ e UR $\left.40 \%\right)$, conforme Anderson \& Baker (1983); Ross (1986); Popinigis (1985). Nessas condições, por outro lado, a manutenção da viabilidade da maioria dos fungos fitopatogênicos é favorecida, comprometendo a qualidade sanitária das sementes, conforme verificado por Oliveira et al. (1997). 
TABELA 3 - Porcentagens de fungos detectados em sementes de milho do lote 3 , armazenadas durante 12 meses em câmara fria e em condição ambiente.

\begin{tabular}{|c|c|c|c|c|c|c|c|c|c|}
\hline \multirow{2}{*}{ Fungo } & \multirow{2}{*}{$\begin{array}{l}\text { Local } \\
\text { armaz. }\end{array}$} & \multicolumn{7}{|c|}{ Mês de armazenamento } & \multirow{2}{*}{ Média } \\
\hline & & 0 & 2 & 4 & 6 & 8 & 10 & 12 & \\
\hline \multirow[t]{2}{*}{ Alternaria alternata } & $\mathrm{CF}^{1}$ & $5 \mathrm{aA}$ & $1 \mathrm{cB}$ & $2 \mathrm{bA}$ & $0 \mathrm{~dB}$ & $0 \mathrm{dA}$ & $0 \mathrm{dA}$ & $0 \mathrm{dA}$ & $1,14 \mathrm{~A}$ \\
\hline & Amb. ${ }^{2}$ & $5 \mathrm{aA}$ & $5 \mathrm{aA}$ & $4 \mathrm{bA}$ & $4 \mathrm{bA}$ & $2 \mathrm{cA}$ & $1 \mathrm{dA}$ & 0 eA & $3,00 \mathrm{~A}$ \\
\hline \multirow[t]{2}{*}{ Aspergillus spp. } & $\mathrm{CF}$ & $9 \mathrm{abA}$ & $10 \mathrm{aA}$ & $10 \mathrm{aA}$ & $8 \mathrm{cB}$ & $9 \mathrm{abB}$ & $9 \mathrm{abB}$ & $9 \mathrm{abB}$ & $9,14 \mathrm{~B}$ \\
\hline & Amb. & $9 \mathrm{dA}$ & $11 \mathrm{dA}$ & $12 \mathrm{cdA}$ & $17 \mathrm{cA}$ & $23 \mathrm{bA}$ & $26 \mathrm{abA}$ & $31 \mathrm{aA}$ & $18,43 \mathrm{~A}$ \\
\hline \multirow[t]{2}{*}{ Bipolaris maydis } & $\mathrm{CF}$ & $2 \mathrm{aA}$ & $1 \mathrm{bA}$ & $2 \mathrm{aA}$ & $1 \mathrm{bA}$ & $1 \mathrm{bA}$ & $1 \mathrm{bA}$ & $1 \mathrm{bA}$ & $1,28 \mathrm{~A}$ \\
\hline & Amb. & $2 \mathrm{aA}$ & $1 \mathrm{bA}$ & $1 \mathrm{bA}$ & $0 \mathrm{cA}$ & $0 \mathrm{cA}$ & $0 \mathrm{cA}$ & $0 \mathrm{cA}$ & $0,57 \mathrm{~A}$ \\
\hline \multirow[t]{2}{*}{ Cephalosporium acremonium } & $\mathrm{CF}$ & 26 aA & $17 \mathrm{bA}$ & $16 \mathrm{bcA}$ & $18 \mathrm{bA}$ & $12 \mathrm{cdA}$ & $12 \mathrm{cdA}$ & $11 \mathrm{dA}$ & $16,00 \mathrm{~A}$ \\
\hline & Amb. & 26 aA & $12 \mathrm{bB}$ & $8 \mathrm{bcB}$ & $7 \mathrm{cdB}$ & $5 \mathrm{cdB}$ & $3 \mathrm{~dB}$ & $3 \mathrm{~dB}$ & $9,14 \mathrm{~B}$ \\
\hline \multirow[t]{2}{*}{ Cladosporium herbarum } & $\mathrm{CF}$ & $14 \mathrm{aA}$ & $10 \mathrm{abA}$ & $8 \mathrm{bcA}$ & $8 \mathrm{bcA}$ & $5 \mathrm{cdA}$ & $3 \mathrm{dA}$ & $3 \mathrm{dA}$ & $5,43 \mathrm{~A}$ \\
\hline & Amb. & $14 \mathrm{aA}$ & $5 \mathrm{bcB}$ & $5 \mathrm{bcA}$ & $6 \mathrm{bA}$ & $2 \mathrm{cA}$ & $3 \mathrm{bcA}$ & $2 \mathrm{cA}$ & $5,29 \mathrm{~A}$ \\
\hline \multirow[t]{2}{*}{ Curvularia spp. } & $\mathrm{CF}$ & 0 & 0 & 1 & 0 & 1 & 0 & 0 & 0,29 \\
\hline & Amb. & 0 & 0 & 0 & 2 & 1 & 1 & 0 & 0,57 \\
\hline \multirow[t]{2}{*}{ Fusarium moniliforme } & $\mathrm{CF}$ & 28 aA & 28 aA & $27 \mathrm{abA}$ & $27 a b A$ & $26 a b A$ & $25 \mathrm{bA}$ & $25 \mathrm{bA}$ & $26,57 \mathrm{~A}$ \\
\hline & Amb. & 28 aA & $19 \mathrm{bB}$ & $21 \mathrm{abB}$ & 15 bB & $13 \mathrm{bcB}$ & $5 \mathrm{cdB}$ & $2 \mathrm{~dB}$ & $14,71 \mathrm{~B}$ \\
\hline \multirow[t]{2}{*}{ Fusarium spp. } & CF & 0 & 0 & 2 & 0 & 0 & 0 & 0 & 0,29 \\
\hline & Amb. & 0 & 0 & 2 & 2 & 0 & 1 & 0 & 0,71 \\
\hline \multirow[t]{2}{*}{ Penicillium spp. } & $\mathrm{CF}$ & 38 abcA & $36 \mathrm{bcB}$ & $35 \mathrm{cB}$ & $39 \mathrm{abcB}$ & $42 \mathrm{aB}$ & $40 \mathrm{abcB}$ & $41 \mathrm{abB}$ & $38,71 \mathrm{~B}$ \\
\hline & Amb. & $38 \mathrm{dA}$ & $43 \mathrm{cdA}$ & $47 \mathrm{bcA}$ & $49 \mathrm{bcA}$ & $52 a b A$ & $55 \mathrm{abA}$ & $59 \mathrm{aA}$ & $49,00 \mathrm{~A}$ \\
\hline \multirow[t]{2}{*}{ Rhizoctonia solani } & $\mathrm{CF}$ & 5 aA & $6 \mathrm{aA}$ & $2 \mathrm{bA}$ & $0 \mathrm{cA}$ & $0 \mathrm{cA}$ & $0 \mathrm{cA}$ & $0 \mathrm{cA}$ & $1,86 \mathrm{~A}$ \\
\hline & Amb. & $5 \mathrm{aA}$ & $2 \mathrm{bB}$ & $0 \mathrm{cA}$ & $0 \mathrm{cA}$ & $0 \mathrm{cA}$ & $0 \mathrm{cA}$ & $0 \mathrm{cA}$ & $1,00 \mathrm{~A}$ \\
\hline \multirow[t]{2}{*}{ Rhizopus spp. } & $\mathrm{CF}$ & $20 \mathrm{bA}$ & $22 \mathrm{abA}$ & $24 a b A$ & $20 \mathrm{bA}$ & $25 \mathrm{aA}$ & $21 \mathrm{abA}$ & $20 \mathrm{bA}$ & $21,71 \mathrm{~A}$ \\
\hline & Amb. & $20 \mathrm{aA}$ & $23 \mathrm{aA}$ & $18 \mathrm{abB}$ & $18 \mathrm{abA}$ & $10 \mathrm{cB}$ & $16 \mathrm{abcB}$ & $13 \mathrm{bcB}$ & $16,86 \mathrm{~B}$ \\
\hline \multirow[t]{2}{*}{ Trichoderma spp. } & $\mathrm{CF}$ & $1 \mathrm{cA}$ & $2 \mathrm{bcA}$ & $2 \mathrm{bcA}$ & $2 \mathrm{bcA}$ & $5 a b A$ & 6 aA & $9 a b A$ & $3,86 \mathrm{~A}$ \\
\hline & Amb. & $1 \mathrm{bA}$ & $1 \mathrm{bA}$ & $1 \mathrm{bA}$ & $1 \mathrm{bA}$ & $2 \mathrm{bB}$ & $5 a b A$ & $8 \mathrm{aA}$ & $2,71 \mathrm{~A}$ \\
\hline
\end{tabular}

${ }^{1} \mathrm{CF}=$ Câmara fria; ${ }^{2} \mathrm{Amb} .=$ Ambiente. Letras minúsculas iguais nas linhas e maiúsculas nas colunas, para cada fungo, não apresentam diferença estatística (Tukey 5\%).

TABELA 4 - Porcentagens de fungos detectados em sementes de milho do lote 4, armazenadas durante 12 meses em câmara fria e em condição ambiente.

\begin{tabular}{|c|c|c|c|c|c|c|c|c|c|}
\hline \multirow{2}{*}{ Fungo } & \multirow{2}{*}{$\begin{array}{l}\text { Local } \\
\text { armaz. }\end{array}$} & \multicolumn{7}{|c|}{ Mês de armazenamento } & \multirow{2}{*}{ Média } \\
\hline & & 0 & 2 & 4 & 6 & 8 & 10 & 12 & \\
\hline \multirow[t]{2}{*}{ Alternaria alternata } & $\mathrm{CF}^{1}$ & $1 \mathrm{aA}$ & $1 \mathrm{aB}$ & $0 \mathrm{bB}$ & $0 \mathrm{bA}$ & $0 \mathrm{bA}$ & $0 \mathrm{bA}$ & $0 \mathrm{bA}$ & $0,29 \mathrm{~A}$ \\
\hline & Amb. $^{2}$ & $1 \mathrm{cA}$ & $5 \mathrm{aA}$ & $4 \mathrm{abA}$ & $2 \mathrm{bcA}$ & $0 \mathrm{cA}$ & $0 \mathrm{cA}$ & $0 \mathrm{cA}$ & $1,71 \mathrm{~A}$ \\
\hline \multirow[t]{2}{*}{ Aspergillus spp. } & $\mathrm{CF}$ & $15 \mathrm{aA}$ & $13 \mathrm{abcB}$ & $9 \mathrm{bcB}$ & $10 \mathrm{abcB}$ & $8 \mathrm{cB}$ & $11 \mathrm{abcB}$ & $10 \mathrm{abcB}$ & $10,86 \mathrm{~B}$ \\
\hline & Amb. & $15 \mathrm{cA}$ & 18 bcA & $17 \mathrm{bcA}$ & 21 abcA & $24 \mathrm{abA}$ & $28 \mathrm{aA}$ & $28 \mathrm{aA}$ & $21,57 \mathrm{~A}$ \\
\hline \multirow[t]{2}{*}{ Bipolaris maydis } & $\mathrm{CF}$ & $0 \mathrm{bA}$ & $1 \mathrm{abA}$ & $2 \mathrm{aA}$ & $1 \mathrm{abA}$ & $1 \mathrm{abA}$ & $1 \mathrm{abA}$ & $1 \mathrm{abA}$ & $1,00 \mathrm{~A}$ \\
\hline & Amb. & $0 \mathrm{bA}$ & $1 \mathrm{abA}$ & $1 \mathrm{abA}$ & $0 \mathrm{bA}$ & $2 \mathrm{aA}$ & $0 \mathrm{bA}$ & $0 \mathrm{bA}$ & $0,57 \mathrm{~A}$ \\
\hline \multirow[t]{2}{*}{ Cephalosporium acremonium } & $\mathrm{CF}$ & 35 aA & 26 bB & $19 \mathrm{cA}$ & $14 \mathrm{cdA}$ & $10 \mathrm{de} A$ & $6 \mathrm{eA}$ & $5 \mathrm{eA}$ & $16,43 \mathrm{~A}$ \\
\hline & Amb. & 35 aA & $33 \mathrm{bA}$ & $16 \mathrm{cA}$ & $9 \mathrm{~dB}$ & $6 \mathrm{de} A$ & $3 \mathrm{de} A$ & $2 \mathrm{eA}$ & $14,86 \mathrm{~A}$ \\
\hline \multirow[t]{2}{*}{ Cladosporium herbarum } & $\mathrm{CF}$ & $2 \mathrm{aA}$ & $0 \mathrm{bA}$ & $2 \mathrm{aA}$ & $2 \mathrm{aA}$ & $0 \mathrm{bA}$ & $0 \mathrm{bA}$ & $2 \mathrm{aA}$ & $1,14 \mathrm{~A}$ \\
\hline & Amb. & $2 a b A$ & 3 aA & $3 a A$ & $2 \mathrm{abA}$ & $2 a b A$ & $2 a b A$ & $3 \mathrm{aA}$ & $2,43 \mathrm{~A}$ \\
\hline \multirow[t]{2}{*}{ Fusarium moniliforme } & $\mathrm{CF}$ & $38 \mathrm{aA}$ & $38 \mathrm{aA}$ & $35 \mathrm{aA}$ & $31 \mathrm{aA}$ & $21 \mathrm{bA}$ & $13 \mathrm{cA}$ & $15 \mathrm{bcA}$ & $27,29 \mathrm{~A}$ \\
\hline & Amb. & $38 \mathrm{aA}$ & $25 \mathrm{bB}$ & $18 \mathrm{bB}$ & $16 \mathrm{bcB}$ & $17 \mathrm{bcB}$ & $10 \mathrm{cdA}$ & $6 \mathrm{~dB}$ & $18,57 \mathrm{~B}$ \\
\hline \multirow[t]{2}{*}{ Fusarium spp. } & $\mathrm{CF}$ & $0 \mathrm{cA}$ & $0 \mathrm{cA}$ & $4 \mathrm{aA}$ & $2 \mathrm{bA}$ & $0 \mathrm{cA}$ & $1 \mathrm{bcA}$ & $0 \mathrm{cA}$ & $1,00 \mathrm{~A}$ \\
\hline & Amb. & $0 \mathrm{bA}$ & $3 \mathrm{aA}$ & $2 a b A$ & $0 \mathrm{bA}$ & $2 a b A$ & $0 \mathrm{bA}$ & $0 \mathrm{bA}$ & $1,00 \mathrm{~A}$ \\
\hline \multirow[t]{2}{*}{ Gonatobotrys spp. } & $\mathrm{CF}$ & $5 \mathrm{aA}$ & $1 \mathrm{bA}$ & $0 \mathrm{bA}$ & $1 \mathrm{bA}$ & $1 \mathrm{bA}$ & $0 \mathrm{bA}$ & $0 \mathrm{bA}$ & $1,14 \mathrm{~A}$ \\
\hline & Amb. & $5 \mathrm{aA}$ & $3 \mathrm{bA}$ & $2 \mathrm{cdA}$ & $4 a b A$ & $2 \mathrm{cdA}$ & $0 \mathrm{dA}$ & $0 \mathrm{dA}$ & $2,29 \mathrm{~A}$ \\
\hline \multirow[t]{2}{*}{ Penicillium spp. } & $\mathrm{CF}$ & $28 a b c A$ & $25 \mathrm{cA}$ & $24 \mathrm{cB}$ & $27 \mathrm{bcB}$ & $31 \mathrm{abA}$ & $33 \mathrm{aB}$ & $33 \mathrm{aB}$ & $28,71 \mathrm{~B}$ \\
\hline & Amb. & $28 \mathrm{cA}$ & $28 \mathrm{cA}$ & $30 \mathrm{cA}$ & $34 \mathrm{bcA}$ & $34 \mathrm{bcA}$ & $39 a b A$ & $41 \mathrm{aA}$ & $33,43 \mathrm{~A}$ \\
\hline \multirow[t]{2}{*}{ Rhizoctonia solani } & $\mathrm{CF}$ & 3 & 2 & 0 & 0 & 0 & 0 & 0 & 0,71 \\
\hline & Amb. & 3 & 1 & 0 & 0 & 0 & 0 & 0 & 0,57 \\
\hline \multirow[t]{2}{*}{ Rhizopus spp. } & $\mathrm{CF}$ & $0 \mathrm{cA}$ & $0 \mathrm{cA}$ & $0 \mathrm{cA}$ & $0 \mathrm{cA}$ & $5 \mathrm{bA}$ & $6 \mathrm{abA}$ & $8 \mathrm{aA}$ & $2,71 \mathrm{~A}$ \\
\hline & Amb. & $0 \mathrm{cA}$ & $0 \mathrm{cA}$ & $0 \mathrm{cA}$ & $0 \mathrm{cA}$ & $3 a b A$ & $4 \mathrm{aA}$ & $2 \mathrm{bB}$ & $1,29 \mathrm{~A}$ \\
\hline \multirow[t]{2}{*}{ Trichoderma spp. } & $\mathrm{CF}$ & 0 & 0 & 0 & 6 & 4 & 1 & 2 & 1,86 \\
\hline & Amb. & 0 & 0 & 0 & 0 & 0 & 0 & 3 & 0,43 \\
\hline
\end{tabular}

${ }^{1} \mathrm{CF}=$ Câmara fria; ${ }^{2} \mathrm{Amb} .=$ Ambiente. Letras minúsculas iguais nas linhas e maiúsculas nas colunas, para cada fungo, não apresentam diferença estatística (Tukey $5 \%$ ). 
TABELA 5 - Porcentagens de fungos detectados em sementes de milho do lote 5, armazenadas durante 12 meses em câmara fria e em condição ambiente.

\begin{tabular}{|c|c|c|c|c|c|c|c|c|c|}
\hline \multirow{2}{*}{ Fungo } & \multirow{2}{*}{ Local armaz. } & \multicolumn{7}{|c|}{ Mês de armazenamento } & \multirow{2}{*}{ Média } \\
\hline & & 0 & 2 & 4 & 6 & 8 & 10 & 12 & \\
\hline \multirow[t]{2}{*}{ Alternaria alternata } & $\mathrm{CF}^{1}$ & $3 \mathrm{aA}$ & $1 \mathrm{bA}$ & $2 a b A$ & $1 \mathrm{bA}$ & $1 \mathrm{bA}$ & $1 \mathrm{bA}$ & $1 \mathrm{bA}$ & $1,43 \mathrm{~A}$ \\
\hline & Amb. $^{2}$ & $3 \mathrm{bA}$ & $3 \mathrm{bA}$ & $4 a b A$ & $4 \mathrm{aB}$ & $1 \mathrm{cA}$ & $1 \mathrm{cA}$ & $0 \mathrm{dA}$ & $2,29 \mathrm{~A}$ \\
\hline \multirow[t]{2}{*}{ Aspergillus spp. } & CF & $16 \mathrm{abcA}$ & $17 \mathrm{abcA}$ & $12 \mathrm{cB}$ & $19 \mathrm{abB}$ & $13 \mathrm{bcB}$ & $17 \mathrm{abcB}$ & $20 \mathrm{aB}$ & $16,29 \mathrm{~B}$ \\
\hline & Amb. & $16 \mathrm{dA}$ & $19 \mathrm{cdA}$ & $25 \mathrm{bcA}$ & $25 \mathrm{bcA}$ & $29 \mathrm{abA}$ & $33 \mathrm{abA}$ & $35 \mathrm{aA}$ & $26,00 \mathrm{~A}$ \\
\hline \multirow[t]{2}{*}{ Bipolaris maydis } & $\mathrm{CF}$ & $2 \mathrm{aA}$ & $2 \mathrm{aA}$ & $2 a b A$ & $1 \mathrm{bA}$ & $2 \mathrm{aA}$ & $1 \mathrm{bA}$ & $1 \mathrm{bA}$ & $1,57 \mathrm{~A}$ \\
\hline & Amb. & $2 \mathrm{aA}$ & $1 \mathrm{abA}$ & $0 \mathrm{bA}$ & $1 \mathrm{abA}$ & $1 \mathrm{abA}$ & $0 \mathrm{bA}$ & $0 \mathrm{bA}$ & $0,71 \mathrm{~A}$ \\
\hline \multirow[t]{2}{*}{ Cephalosporium acremonium } & $\mathrm{CF}$ & $37 \mathrm{aA}$ & $23 \mathrm{bA}$ & $8 \mathrm{cdB}$ & $12 \mathrm{cA}$ & $9 \mathrm{cdA}$ & $7 \mathrm{cdA}$ & $4 \mathrm{dA}$ & $14,29 \mathrm{~A}$ \\
\hline & Amb. & $37 \mathrm{aA}$ & $22 \mathrm{bA}$ & $15 \mathrm{bcA}$ & $8 \mathrm{cdB}$ & $2 \mathrm{~dB}$ & $3 \mathrm{~dB}$ & $1 \mathrm{dA}$ & $12,57 \mathrm{~A}$ \\
\hline \multirow[t]{2}{*}{ Cladosporium herbarum } & $\mathrm{CF}$ & $11 \mathrm{aA}$ & $5 \mathrm{bA}$ & $5 \mathrm{bA}$ & $2 \mathrm{bcA}$ & $1 \mathrm{cA}$ & $0 \mathrm{cA}$ & $0 \mathrm{cB}$ & $3,43 \mathrm{~A}$ \\
\hline & Amb. & $11 \mathrm{aA}$ & $5 \mathrm{bA}$ & $4 \mathrm{bA}$ & $5 \mathrm{bA}$ & $4 \mathrm{bB}$ & $3 \mathrm{bA}$ & $4 \mathrm{bA}$ & $5,14 \mathrm{~A}$ \\
\hline \multirow[t]{2}{*}{ Curvularia spp. } & $\mathrm{CF}$ & 1 & 1 & 0 & 1 & 0 & 0 & 0 & 0,43 \\
\hline & Amb. & 1 & 2 & 1 & 0 & 0 & 0 & 0 & 0,57 \\
\hline \multirow[t]{2}{*}{ Fusarium moniliforme } & $\mathrm{CF}$ & $47 \mathrm{aA}$ & $42 \mathrm{abA}$ & $44 \mathrm{abA}$ & $42 \mathrm{abA}$ & $39 \mathrm{bcA}$ & $34 \mathrm{cA}$ & $32 \mathrm{cA}$ & $40,00 \mathrm{~A}$ \\
\hline & Amb. & $47 \mathrm{aA}$ & $40 \mathrm{aA}$ & $17 \mathrm{bB}$ & $18 \mathrm{bB}$ & $14 \mathrm{bcB}$ & $9 \mathrm{cB}$ & $6 \mathrm{cB}$ & $21,57 \mathrm{~B}$ \\
\hline \multirow[t]{2}{*}{ Fusarium spp. } & $\mathrm{CF}$ & 1 & 1 & 0 & 1 & 0 & 0 & 0 & 0,43 \\
\hline & Amb. & 0 & 0 & 0 & 0 & 0 & 0 & 0 & 0,00 \\
\hline \multirow[t]{2}{*}{ Penicillium spp. } & $\mathrm{CF}$ & $41 \mathrm{abcA}$ & $39 \mathrm{bcB}$ & $37 \mathrm{cB}$ & $35 \mathrm{cB}$ & $39 \mathrm{bcB}$ & $45 \mathrm{abB}$ & $46 \mathrm{aB}$ & $40,29 \mathrm{~B}$ \\
\hline & Amb. & $41 \mathrm{dA}$ & $43 \mathrm{cdA}$ & $44 \mathrm{cdA}$ & $49 \mathrm{bcA}$ & $52 \mathrm{aA}$ & $59 \mathrm{aA}$ & $63 \mathrm{aA}$ & $50,14 \mathrm{~A}$ \\
\hline \multirow[t]{2}{*}{ Rhizopus spp. } & $\mathrm{CF}$ & $4 \mathrm{aA}$ & $3 a b A$ & $4 \mathrm{aA}$ & $2 \mathrm{bcA}$ & $0 \mathrm{cB}$ & $2 \mathrm{bA}$ & $2 \mathrm{bA}$ & $2,43 \mathrm{~A}$ \\
\hline & Amb. & $4 a b A$ & $0 \mathrm{cA}$ & $0 \mathrm{cB}$ & $5 \mathrm{aA}$ & $6 \mathrm{aA}$ & $2 \mathrm{bcA}$ & $1 \mathrm{cA}$ & $2,57 \mathrm{~A}$ \\
\hline \multirow[t]{2}{*}{ Trichoderma spp. } & $\mathrm{CF}$ & 0 & 0 & 0 & 0 & 0 & 0 & 4 & 0,57 \\
\hline & Amb. & 0 & 1 & 0 & 4 & 0 & 1 & 1 & 1,00 \\
\hline
\end{tabular}

${ }^{1} \mathrm{CF}=$ Câmara fria; ${ }^{2} \mathrm{Amb} .=$ Ambiente. Letras minúsculas iguais nas linhas e maiúsculas nas colunas, para cada fungo, não apresentam diferença estatística (Tukey 5\%).

TABELA 6 - Porcentagens de fungos detectados em sementes de milho do lote 6, armazenadas durante 12 meses em câmara fria e em condição ambiente.

\begin{tabular}{|c|c|c|c|c|c|c|c|c|c|}
\hline \multirow{2}{*}{ Fungo } & \multirow{2}{*}{ Local armaz. } & \multicolumn{7}{|c|}{ Mês de armazenamento } & \multirow{2}{*}{ Média } \\
\hline & & 0 & 2 & 4 & 6 & 8 & 10 & 12 & \\
\hline \multirow[t]{2}{*}{ Alternaria alternata } & $\mathrm{CF}^{1}$ & 1 & 2 & 1 & 0 & 0 & 0 & 0 & 0,57 \\
\hline & Amb. ${ }^{2}$ & 0 & 3 & 0 & 0 & 0 & 0 & 0 & 0,43 \\
\hline \multirow[t]{2}{*}{ Aspergillus spp. } & CF & $8 \mathrm{aA}$ & $5 \mathrm{bB}$ & $5 \mathrm{bB}$ & $5 \mathrm{bB}$ & $4 \mathrm{bB}$ & $6 \mathrm{abB}$ & $5 \mathrm{bB}$ & $5,43 \mathrm{~B}$ \\
\hline & Amb. & $8 \mathrm{cA}$ & 12 bcA & $18 \mathrm{aA}$ & 19 aA & $17 \mathrm{aA}$ & $16 \mathrm{abA}$ & $18 \mathrm{aA}$ & $15,43 \mathrm{~A}$ \\
\hline \multirow[t]{2}{*}{ Bipolaris maydis } & $\mathrm{CF}$ & 0 & 1 & 0 & 0 & 0 & 0 & 0 & 0,14 \\
\hline & Amb. & 0 & 0 & 0 & 0 & 0 & 0 & 0 & 0,00 \\
\hline \multirow[t]{2}{*}{ Cephalosporium acremonium } & CF & $13 \mathrm{aA}$ & $7 \mathrm{bA}$ & $7 \mathrm{bA}$ & $4 \mathrm{bcB}$ & $3 \mathrm{bcA}$ & $2 \mathrm{bA}$ & $3 \mathrm{bcA}$ & $5,57 \mathrm{~A}$ \\
\hline & Amb. & $13 \mathrm{aA}$ & $9 \mathrm{bA}$ & 7 bcA & $9 \mathrm{bA}$ & $4 \mathrm{~cd} A$ & $3 \mathrm{~cd} A$ & $1 \mathrm{dA}$ & $6,57 \mathrm{~A}$ \\
\hline \multirow[t]{2}{*}{ Cladosporium herbarum } & $\mathrm{CF}$ & $8 \mathrm{aA}$ & $5 a b A$ & $6 \mathrm{aA}$ & $2 \mathrm{bcA}$ & $1 \mathrm{cA}$ & $1 \mathrm{cA}$ & $2 \mathrm{bcA}$ & $3,57 \mathrm{~A}$ \\
\hline & Amb. & 8 aA & $6 a b A$ & $3 \mathrm{bA}$ & $3 \mathrm{bA}$ & $4 \mathrm{bA}$ & $3 \mathrm{bA}$ & $4 \mathrm{bA}$ & $4,43 \mathrm{~A}$ \\
\hline \multirow[t]{2}{*}{ Colletotrichum graminicola } & $C F$ & 0 & 1 & 0 & 0 & 0 & 0 & 0 & 0,14 \\
\hline & Amb. & 0 & 0 & 0 & 0 & 0 & 0 & 0 & 0,00 \\
\hline \multirow[t]{2}{*}{ Fusarium moniliforme } & $\mathrm{CF}$ & $84 \mathrm{aA}$ & $80 \mathrm{aA}$ & $79 a b A$ & $79 a b A$ & $77 \mathrm{abA}$ & $73 \mathrm{bcA}$ & $70 \mathrm{cA}$ & $77,42 \mathrm{~A}$ \\
\hline & Amb. & $84 \mathrm{aA}$ & 76 bB & $46 \mathrm{cB}$ & $39 \mathrm{cdB}$ & $29 \mathrm{~dB}$ & $20 \mathrm{eB}$ & $14 \mathrm{eB}$ & $44,00 \mathrm{~B}$ \\
\hline \multirow[t]{2}{*}{ Fusarium spp. } & CF & 6 aA & $5 a b A$ & $3 a b c A$ & $3 a b c A$ & $2 \mathrm{bcA}$ & $2 \mathrm{bcA}$ & $1 \mathrm{cA}$ & $3,14 \mathrm{~A}$ \\
\hline & Amb. & $6 \mathrm{aA}$ & $3 \mathrm{bA}$ & $2 \mathrm{bcA}$ & $1 \mathrm{cA}$ & $1 \mathrm{cA}$ & $0 \mathrm{cA}$ & $0 \mathrm{cA}$ & $1,86 \mathrm{~A}$ \\
\hline \multirow[t]{2}{*}{ Penicillium spp. } & $\mathrm{CF}$ & $47 \mathrm{abA}$ & $44 \mathrm{bA}$ & $44 \mathrm{bB}$ & $47 \mathrm{abB}$ & $49 \mathrm{abB}$ & $48 \mathrm{abB}$ & $51 \mathrm{aB}$ & $41,43 \mathrm{~B}$ \\
\hline & Amb. & $47 \mathrm{dA}$ & $47 \mathrm{dA}$ & $53 \mathrm{cdA}$ & $56 \mathrm{bcA}$ & $59 \mathrm{bcA}$ & $62 \mathrm{abA}$ & $65 \mathrm{aA}$ & $55,57 \mathrm{~A}$ \\
\hline \multirow[t]{2}{*}{ Rhizoctonia solani } & CF & 1 & 0 & 0 & 0 & 1 & 0 & 2 & 0,57 \\
\hline & Amb. & 1 & 0 & 0 & 1 & 1 & 1 & 0 & 0,57 \\
\hline \multirow[t]{2}{*}{ Rhizopus spp. } & $\mathrm{CF}$ & $0 \mathrm{cA}$ & $0 \mathrm{cA}$ & $0 \mathrm{cA}$ & $4 \mathrm{aB}$ & $3 a b B$ & $1 \mathrm{bB}$ & $2 a b B$ & $1,14 \mathrm{~B}$ \\
\hline & Amb. & $0 \mathrm{cA}$ & $0 \mathrm{cA}$ & $0 \mathrm{cA}$ & $9 a b A$ & $7 \mathrm{bA}$ & $12 \mathrm{aA}$ & $10 \mathrm{abA}$ & $5,43 \mathrm{~A}$ \\
\hline \multirow[t]{2}{*}{ Trichoderma spp. } & $\mathrm{CF}$ & $0 \mathrm{bA}$ & $0 \mathrm{bA}$ & $0 \mathrm{bA}$ & $0 \mathrm{bB}$ & $5 \mathrm{aA}$ & $1 \mathrm{bA}$ & $1 \mathrm{bA}$ & $1,00 \mathrm{~A}$ \\
\hline & Amb. & $0 \mathrm{ba}$ & $0 \mathrm{cA}$ & $0 \mathrm{cA}$ & 7 aA & $3 \mathrm{bA}$ & $0 \mathrm{cA}$ & $4 \mathrm{bA}$ & $2,00 \mathrm{~A}$ \\
\hline
\end{tabular}

${ }^{1} \mathrm{CF}=$ Câmara fria; ${ }^{2} \mathrm{Amb} .=$ Ambiente. Letras minúsculas iguais nas linhas e maiúsculas nas colunas, para cada fungo, não apresentam diferença estatística (Tukey $5 \%$ ). 
TABELA 7 - Porcentagens de fungos detectados em sementes de milho do lote 7, armazenadas durante 12 meses em câmara fria e em condição ambiente.

\begin{tabular}{|c|c|c|c|c|c|c|c|c|c|}
\hline \multirow{2}{*}{ Fungo } & \multirow{2}{*}{ Local armaz. } & \multicolumn{7}{|c|}{ Mês de armazenamento } & \multirow{2}{*}{ Média } \\
\hline & & 0 & 2 & 4 & 6 & 8 & 10 & 12 & \\
\hline \multirow[t]{2}{*}{ Alternaria alternata } & $\mathrm{CF}^{1}$ & $2 \mathrm{aA}$ & $1 \mathrm{abB}$ & $2 \mathrm{aB}$ & $1 \mathrm{abA}$ & $0 \mathrm{bA}$ & $0 \mathrm{bB}$ & $0 \mathrm{bA}$ & $0,86 \mathrm{~A}$ \\
\hline & $\mathrm{Amb}^{2}$ & $2 \mathrm{bA}$ & $3 a b A$ & $4 \mathrm{aA}$ & $0 \mathrm{cA}$ & $0 \mathrm{cA}$ & $2 \mathrm{bA}$ & $0 \mathrm{cA}$ & $1,57 \mathrm{~A}$ \\
\hline \multirow[t]{2}{*}{ Aspergillus spp. } & CF & $13 \mathrm{abA}$ & $10 \mathrm{cB}$ & $14 \mathrm{aA}$ & $10 \mathrm{cB}$ & $13 \mathrm{abB}$ & $9 \mathrm{cB}$ & $12 a b B$ & $11,57 \mathrm{~B}$ \\
\hline & Amb. & $13 \mathrm{cA}$ & $17 \mathrm{bcA}$ & $17 \mathrm{bcA}$ & $19 \mathrm{bcA}$ & $19 \mathrm{bcA}$ & $23 \mathrm{abA}$ & $27 \mathrm{aA}$ & $19,29 \mathrm{~A}$ \\
\hline \multirow[t]{2}{*}{ Bipolaris maydis } & $\mathrm{CF}$ & $2 \mathrm{aA}$ & $1 \mathrm{bA}$ & $1 \mathrm{bA}$ & $1 \mathrm{bA}$ & $2 \mathrm{aA}$ & $1 \mathrm{bA}$ & $2 \mathrm{aA}$ & $1,43 \mathrm{~A}$ \\
\hline & Amb. & $1 \mathrm{aA}$ & $1 \mathrm{aA}$ & $1 \mathrm{aA}$ & $1 \mathrm{aA}$ & $1 \mathrm{aA}$ & $0 \mathrm{bA}$ & $0 \mathrm{bB}$ & $0,71 \mathrm{~A}$ \\
\hline \multirow[t]{2}{*}{ Cephalosporium acremonium } & CF & 26 aA & $23 \mathrm{aA}$ & $25 \mathrm{aA}$ & $15 \mathrm{bA}$ & $14 \mathrm{bA}$ & $13 \mathrm{bA}$ & $12 \mathrm{bA}$ & $18,29 \mathrm{~A}$ \\
\hline & Amb. & 26 aA & 20 aA & $13 \mathrm{bB}$ & $9 \mathrm{bcB}$ & $5 \mathrm{cdB}$ & $3 \mathrm{~dB}$ & $2 \mathrm{~dB}$ & $11,14 \mathrm{~B}$ \\
\hline \multirow[t]{2}{*}{ Cladosporium herbarum } & CF & $5 \mathrm{aA}$ & $5 \mathrm{aA}$ & $2 \mathrm{bB}$ & $1 \mathrm{bB}$ & $1 \mathrm{bB}$ & $2 \mathrm{bB}$ & $0 \mathrm{bB}$ & $2,29 \mathrm{~B}$ \\
\hline & Amb. & $5 \mathrm{bA}$ & $7 \mathrm{abA}$ & $8 \mathrm{abA}$ & $9 \mathrm{aA}$ & $9 \mathrm{aA}$ & $8 \mathrm{abA}$ & $8 a b A$ & $7,71 \mathrm{~A}$ \\
\hline \multirow[t]{2}{*}{ Curvularia spp. } & $\mathrm{CF}$ & $0 \mathrm{bA}$ & $0 \mathrm{bA}$ & $1 \mathrm{bA}$ & $2 \mathrm{abA}$ & $0 \mathrm{bA}$ & $4 \mathrm{aA}$ & $0 \mathrm{bA}$ & $1,00 \mathrm{~A}$ \\
\hline & Amb. & $0 \mathrm{bA}$ & $2 \mathrm{aA}$ & $2 \mathrm{aA}$ & $0 \mathrm{bA}$ & $0 \mathrm{bA}$ & $0 \mathrm{bB}$ & $0 \mathrm{bA}$ & $1,43 \mathrm{~A}$ \\
\hline \multirow[t]{2}{*}{ Epicoccum spp. } & $\mathrm{CF}$ & $4 \mathrm{aA}$ & $0 \mathrm{cA}$ & $0 \mathrm{cA}$ & $0 \mathrm{cA}$ & $1 \mathrm{bA}$ & $0 \mathrm{cA}$ & $0 \mathrm{cA}$ & $0,71 \mathrm{~A}$ \\
\hline & Amb. & $4 \mathrm{aA}$ & $0 \mathrm{cA}$ & $0 \mathrm{cA}$ & $2 \mathrm{bA}$ & $2 \mathrm{bA}$ & $0 \mathrm{cA}$ & $0 \mathrm{cA}$ & $1,14 \mathrm{~A}$ \\
\hline \multirow[t]{2}{*}{ Fusarium moniliforme } & $\mathrm{CF}$ & $34 \mathrm{aA}$ & $34 \mathrm{aA}$ & $32 \mathrm{abA}$ & $30 \mathrm{bA}$ & $29 \mathrm{bA}$ & $31 \mathrm{abA}$ & $30 \mathrm{bA}$ & $31,43 \mathrm{~A}$ \\
\hline & Amb. & $34 \mathrm{aA}$ & $22 \mathrm{bA}$ & $17 \mathrm{bcB}$ & $14 \mathrm{cdB}$ & $11 \mathrm{cdeB}$ & $8 \mathrm{deB}$ & $4 \mathrm{eB}$ & $15,71 \mathrm{~B}$ \\
\hline \multirow[t]{2}{*}{ Nigrospora spp. } & CF & 0 & 0 & 0 & 0 & 2 & 0 & 0 & 2,00 \\
\hline & Amb. & 0 & 0 & 0 & 0 & 0 & 2 & 0 & 2,00 \\
\hline \multirow[t]{2}{*}{ Penicillium spp. } & CF & $33 \mathrm{abA}$ & $31 \mathrm{abB}$ & $30 \mathrm{bB}$ & $32 \mathrm{abB}$ & $35 \mathrm{aB}$ & $34 \mathrm{aB}$ & $34 \mathrm{aB}$ & $32,71 \mathrm{~B}$ \\
\hline & Amb. & $33 \mathrm{dA}$ & $36 \mathrm{cdA}$ & $39 \mathrm{bcdA}$ & $44 \mathrm{bcA}$ & $42 \mathrm{bcA}$ & $49 a b A$ & $53 \mathrm{aA}$ & $42,29 \mathrm{~A}$ \\
\hline \multirow[t]{2}{*}{ Rhizoctonia solani } & $\mathrm{CF}$ & 2 & 1 & 0 & 0 & 0 & 0 & 0 & 0,43 \\
\hline & Amb. & 2 & 2 & 0 & 0 & 0 & 0 & 0 & 0,57 \\
\hline \multirow[t]{2}{*}{ Rhizopus spp. } & $\mathrm{CF}$ & $0 \mathrm{cA}$ & $1 \mathrm{bcA}$ & $0 \mathrm{cA}$ & $7 \mathrm{aA}$ & $3 \mathrm{bA}$ & $3 \mathrm{bA}$ & $6 \mathrm{aA}$ & $2,86 \mathrm{~A}$ \\
\hline & Amb. & $0 \mathrm{bA}$ & $0 \mathrm{bA}$ & $0 \mathrm{bA}$ & $5 \mathrm{aA}$ & $2 \mathrm{bA}$ & $4 \mathrm{aA}$ & $2 \mathrm{bB}$ & $1,71 \mathrm{~A}$ \\
\hline \multirow[t]{2}{*}{ Trichoderma spp. } & CF & 0 & 0 & 2 & 3 & 6 & 0 & 2 & 2,00 \\
\hline & Amb. & 0 & 0 & 0 & 3 & 0 & 0 & 0 & 0,43 \\
\hline
\end{tabular}

${ }^{1} \mathrm{CF}=$ Câmara fria; ${ }^{2} \mathrm{Amb} .=$ Ambiente. Letras minúsculas iguais nas linhas e maiúsculas nas colunas, para cada fungo, não apresentam diferença estatística (Tukey 5\%).

TABELA 8 - Porcentagens de fungos detectados em sementes de milho do lote 8, armazenadas durante 12 meses em câmara fria e em condição ambiente.

\begin{tabular}{|c|c|c|c|c|c|c|c|c|c|}
\hline \multirow{2}{*}{ Fungo } & \multirow{2}{*}{ Local armaz. } & \multicolumn{7}{|c|}{ Mês de armazenamento } & \multirow{2}{*}{ Média } \\
\hline & & 0 & 2 & 4 & 6 & 8 & 10 & 12 & \\
\hline \multirow[t]{2}{*}{ Alternaria alternata } & $\mathrm{CF}^{1}$ & $0 \mathrm{bA}$ & $4 \mathrm{aA}$ & $0 \mathrm{bA}$ & $1 \mathrm{bA}$ & $1 \mathrm{bA}$ & $0 \mathrm{bA}$ & $0 \mathrm{bA}$ & $0,86 \mathrm{~A}$ \\
\hline & $\mathrm{Amb}^{2}$ & $0 \mathrm{bA}$ & $2 \mathrm{aA}$ & $2 \mathrm{aB}$ & $2 \mathrm{aA}$ & $0 \mathrm{bA}$ & $0 \mathrm{bA}$ & $2 \mathrm{aA}$ & $1,14 \mathrm{~A}$ \\
\hline \multirow[t]{2}{*}{ Aspergillus spp. } & CF & $6 a b c A$ & $4 \mathrm{cA}$ & $4 \mathrm{cB}$ & $5 \mathrm{bcB}$ & $7 \mathrm{abB}$ & $6 \mathrm{abcB}$ & $9 \mathrm{aB}$ & $5,87 \mathrm{~B}$ \\
\hline & Amb. & $6 \mathrm{cA}$ & $6 \mathrm{cA}$ & $10 \mathrm{bcA}$ & $14 \mathrm{abcA}$ & $15 a b A$ & $19 \mathrm{aA}$ & $22 \mathrm{aA}$ & $11,29 \mathrm{~A}$ \\
\hline \multirow[t]{2}{*}{ Bipolaris maydis } & $\mathrm{CF}$ & $2 \mathrm{aA}$ & $2 \mathrm{aA}$ & $2 \mathrm{aA}$ & $1 \mathrm{bA}$ & $1 \mathrm{bA}$ & $1 \mathrm{bA}$ & $1 \mathrm{bA}$ & $1,43 \mathrm{~A}$ \\
\hline & Amb. & $2 \mathrm{aA}$ & $2 \mathrm{aA}$ & $1 \mathrm{abA}$ & $1 \mathrm{abA}$ & $0 \mathrm{bA}$ & $1 \mathrm{abA}$ & $0 \mathrm{bA}$ & $1,00 \mathrm{~A}$ \\
\hline \multirow[t]{2}{*}{ Cephalosporium acremonium } & $\mathrm{CF}$ & $19 \mathrm{aA}$ & $15 \mathrm{abA}$ & $11 \mathrm{bcB}$ & $10 \mathrm{bcA}$ & $9 \mathrm{cA}$ & $8 \mathrm{cA}$ & $8 \mathrm{cA}$ & $11,42 \mathrm{~A}$ \\
\hline & Amb. & $19 \mathrm{aA}$ & $18 \mathrm{aA}$ & $15 \mathrm{aA}$ & $9 \mathrm{bA}$ & $7 \mathrm{bA}$ & $8 \mathrm{bA}$ & $2 \mathrm{cB}$ & $11,14 \mathrm{~A}$ \\
\hline \multirow[t]{2}{*}{ Cladosporium herbarum } & CF & $1 \mathrm{abA}$ & $0 \mathrm{bA}$ & $2 \mathrm{aA}$ & $1 \mathrm{abB}$ & $1 \mathrm{abA}$ & $0 \mathrm{bA}$ & $2 \mathrm{aB}$ & $1,00 \mathrm{~A}$ \\
\hline & Amb. & $1 \mathrm{cA}$ & $0 \mathrm{cA}$ & $0 \mathrm{cA}$ & $5 \mathrm{abA}$ & $0 \mathrm{cA}$ & $3 \mathrm{bcA}$ & $7 \mathrm{aA}$ & $2,29 \mathrm{~A}$ \\
\hline \multirow[t]{2}{*}{ Fusarium moniliforme } & $\mathrm{CF}$ & 69 aA & $64 \mathrm{abA}$ & $63 \mathrm{abA}$ & $61 \mathrm{bcA}$ & $60 \mathrm{bcA}$ & $59 \mathrm{bcA}$ & $56 \mathrm{cA}$ & $61,71 \mathrm{~A}$ \\
\hline & Amb. & $69 \mathrm{aA}$ & $56 \mathrm{bB}$ & $42 \mathrm{cB}$ & $40 \mathrm{cB}$ & $31 \mathrm{~dB}$ & $25 \mathrm{~dB}$ & $17 \mathrm{eB}$ & $40,00 \mathrm{~B}$ \\
\hline \multirow[t]{2}{*}{ Penicillium spp. } & CF & 66 aA & $54 \mathrm{cB}$ & $56 \mathrm{cB}$ & $59 \mathrm{bcB}$ & $59 \mathrm{bB}$ & $65 \mathrm{abB}$ & $66 \mathrm{aB}$ & $60,71 \mathrm{~B}$ \\
\hline & Amb. & $66 \mathrm{cA}$ & $65 \mathrm{cA}$ & $65 \mathrm{cA}$ & $71 \mathrm{bcA}$ & $75 \mathrm{abA}$ & $79 a b A$ & $82 \mathrm{aA}$ & $71,86 \mathrm{~A}$ \\
\hline \multirow[t]{2}{*}{ Rhizoctonia solani } & $\mathrm{CF}$ & 0 & 1 & 0 & 0 & 0 & 0 & 0 & 0,14 \\
\hline & Amb. & 0 & 0 & 0 & 0 & 0 & 0 & 0 & 0,00 \\
\hline \multirow[t]{2}{*}{ Rhizopus spp. } & CF & $0 \mathrm{bA}$ & $0 \mathrm{bA}$ & $0 \mathrm{bA}$ & $1 \mathrm{bA}$ & $0 \mathrm{bB}$ & $7 \mathrm{aA}$ & $1 \mathrm{bA}$ & $1,29 \mathrm{~A}$ \\
\hline & Amb. & $0 \mathrm{bA}$ & $2 \mathrm{bA}$ & $2 \mathrm{bA}$ & $0 \mathrm{bA}$ & $5 \mathrm{aA}$ & $4 \mathrm{aA}$ & $0 \mathrm{bA}$ & $1,86 \mathrm{~A}$ \\
\hline \multirow[t]{2}{*}{ Trichoderma spp. } & $\mathrm{CF}$ & 0 & 0 & 0 & 0 & 2 & 4 & 4 & 1,43 \\
\hline & Amb. & 0 & 0 & 0 & 1 & 0 & 2 & 2 & 0,71 \\
\hline
\end{tabular}

${ }^{1} \mathrm{CF}=$ Câmara fria; ${ }^{2} \mathrm{Amb} .=$ Ambiente. Letras minúsculas iguais nas linhas e maiúsculas nas colunas, para cada fungo, não apresentam diferença estatística (Tukey $5 \%$ ). 


\section{AGRADECIMENTO}

Ao Dr. Antônio Augusto do Lago, Pesquisador Científico do Centro de Produção de Material Propagativo, do Instituto Agronômico de Campinas, pela revisão do Abstract.

\section{REFERÊNCIAS BIBLIOGRÁFICAS}

ANDERSON, J.D.; BAKER, J.E. Deterioration of seeds during aging. Phytopathology, v.73, p.321-325, 1983.

BACON, C.W.; HINTON, D.M.; RICHARDSON, M.D. A corn seedling assay for resistance to Fusarium moniliforme. Plant Disease, v.78, p.302-305, 1994.

BERJAK, P. Stored seeds: the problems caused by microorganisms. In: ADVANCED INTERNATIONAL COURSE ON SEED PATHOLOGY, Passo Fundo, 1987. Proceedings. Passo Fundo: EMBRAPA; ABRATES, 1987a. p.93-112.

BERJAK, P. Seed stored problems: our research program. In: ADVANCED INTERNATIONAL COURSE ON SEED PATHOLOGY, Passo Fundo, 1987. Proceedings. Passo Fundo: EMBRAPA; ABRATES, 1987b. p.113-130.

CARVALHO, N.M.; NAKAGAWA, J. Sementes: ciência, tecnologia e produção. 3.ed. Campinas: Fundação Cargill, 1988. 424p.

CASA, R.T.; REIS, E.M.; MEDEIROS, C.A.; MOURA, F.B. Efeito do tratamento de sementes de milho com fungicidas, na proteção de fungos do solo, no Rio Grande do Sul. Fitopatologia Brasileira, v.20, p.633-637, 1995.

CASA, R.T.; REIS, E.M.; ZAMBOLIM, L. Fungos associados à semente de milho produzida nas regiões sul e sudeste do Brasil. Fitopatologia Brasileira, v.23, p.370-373, 1998.

FERNANDES, F.T.; OLIVEIRA, E. Principais doenças na cultura do milho. Sete Lagoas: EMBRAPA, CNPMS, 1997. 80p. (Circular Técnica, 26).

GOULART, A.C.P.; FIALHO, W.F.B. Incidência e controle de Fusarium moniliforme em sementes de milho. Informativo ABRATES, v.9, p.110, 1999

HALFON-MEIRI, A.; SOLEL, Z. Factors affecting seedling blight of sweet corn caused by seedborne Penicillium oxalicum. Plant Disease, v.74, p.36-39, 1990.

LAL, S.P.; KAPOOR, J.N. Succession of fungi in wheat and maize during storage. Indian Phytopathology, v.32, p.101-104, 1979.

LUCA FILHO, O.A. Testes de sanidade de sementes de milho. In: SOAVE, J.; WETZEL, M.M.V.S. (Ed.) Patologia de sementes. Campinas: Fundação Cargill, 1987. p.430-440.

LUZ, W.C. Diagnose e controle das doenças da espiga de milho no Brasil. Passo Fundo: EMBRAPA, CNPT, 1995. 28p. (Circular Técnica, 5).

MACHADO, J.C. Patologia de sementes: fundamentos e aplicações. Brasília: MEC; FAEPE, 1988. 107p.
MERONUCK, R.A. The significance of fungi in cereal grains. Plant Disease, v.71, p.287-291, 1987.

OCHOR, T.E.; TREVATHAN, L.E.; KING, S.B. Relationship of harvest date and host genotype to infection of maize kernels by Fusarium moniliforme. Plant Disease, v.71, p.311-313, 1987.

OLIVEIRA, J.A.; VIEIRA, M.G.G.C.; VON PINHO, E.V.R.; CARVALHO, M.L.M. Comportamento de sementes de milho tratadas com fungicidas antes e após o armazenamento convencional. Revista Brasileira de Sementes, v.19, p.208213, 1997.

PEIXOTO, A.R.; TORRES, S.B.; KARASAWA, N. Qualidade sanitária de sementes de milho produzidas no submédio São Francisco. Revista Brasileira de Sementes, v.20, p.12-15, 1998.

PINTO, N.F.J.A. Patologia de sementes de milho. Sete Lagoas: EMBRAPA, CNPMS, 1998. 44p. (Circular Técnica, 29).

POPINIGIS, F. Fisiologia da semente. 2.ed. Brasília: Pax, 1985. 289p.

REIS, A.C.; REIS, E.M.; CASA, R.T.; FORCELINI, C.A. Erradicação de fungos patogênicos associados a sementes de milho e proteção contra Pythium sp. presente no solo pelo tratamento com fungicidas. Fitopatologia Brasileira, v.20, p.585-590, 1995.

ROSS, E.E. Precepts of successful seed storage. In: HORNER, E.S.; KRAL, D.M.; MICKELSON, S.H. (Ed.) Physiology of seed deterioration. Madison: CSSA, 1986. p.1-25.

TANAKA, M.A.S. Fatores influenciando a germinação de sementes de milho (Zea mays L.) em presença de Fusarium moniliforme Sheldon. Piracicaba, 1976. 113p. Dissertação (Mestrado) - Escola Superior de Agricultura "Luiz de Queiroz", Universidade de São Paulo.

TANAKA, M.A.S.; BALMER, E. Efeito da temperatura e dos microrganismos associados do tombamento na germinação de sementes de milho (Zea mays L.). Fitopatologia Brasileira, v.5, p.87-93, 1980.

TEMPE, J. de. Routine methods for determining the health condition of seeds in the seed testing station. Proceedings of International Seed Testing Association, v.35, p.3-41, 1970.

TUITE, J.; FORSTER, G.H. Control of storage diseases of grain. Annual Review of Phytopathology, v.17, p.343-346, 1979.

TUITE, J.; KOH-KNOX, C; STROSHINE, R.; CANTONE, F.A.; BAUMAN, L.F. Effect of physical damage to corn kernels on the development of Penicillium species and Aspergillus glaucus in storage. Phytopathology, v.75, p.1137-1140, 1985.

WETZEL, M.M.V.S. Fungos de armazenamento. In: SOAVE, J.; WETZEL, M.M.V.S. (Ed.) Patologia de sementes. Campinas: Fundação Cargill, 1987. p.260-275.

$\overline{\text { Recebido em } 04.05 .00}$ 